\title{
Medical Image of the Week: Thoracic Splenosis
}
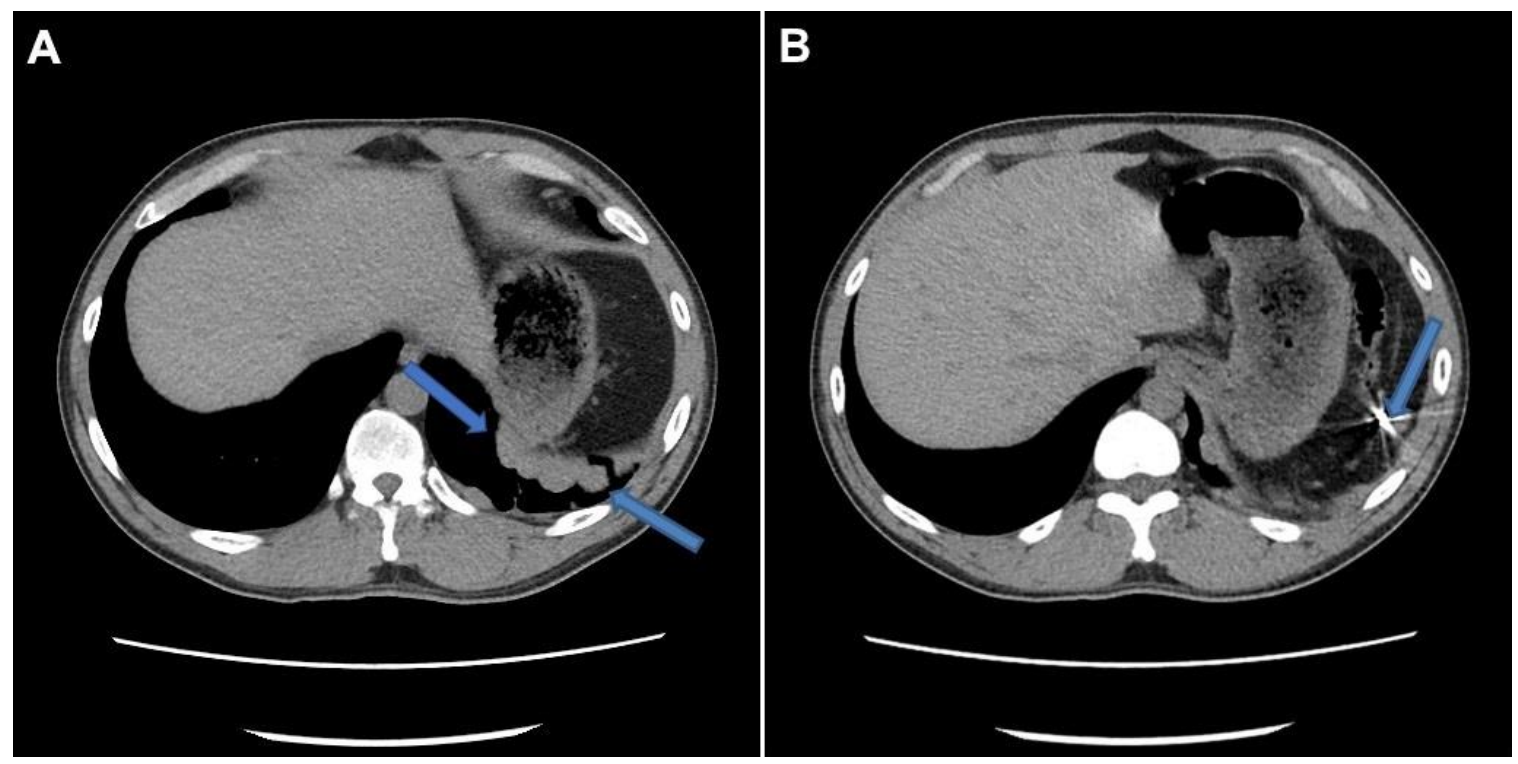

Figure 1. A: Axial CT of the chest without intravenous contrast demonstrates a cluster of soft tissue nodules adjacent to the left posterior hemi-diaphragm (blue arrows). B: Axial CT of the chest without intravenous contrast demonstrates absence of the spleen and a surgical clip (blue arrow) consistent with a prior splenectomy.

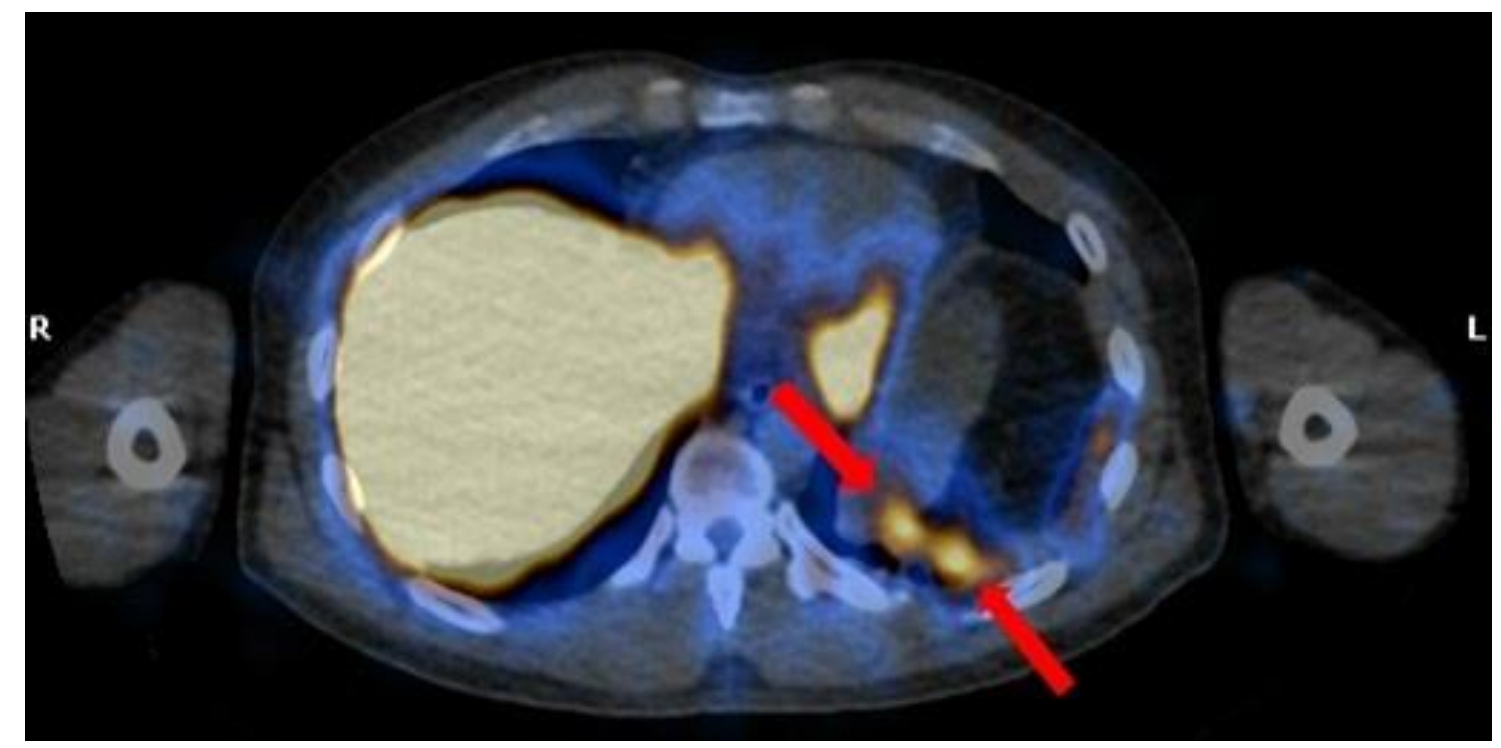

Figure 2. Fused technetium 99m-label sulfur colloid uptake study and axial CT of the chest without intravenous contrast demonstrates uptake of radiotracer in the previously seen soft tissue nodules adjacent to the posterior aspect of the left hemi-diaphragm (red arrows) which confirms the diagnosis of thoracic splenosis. 
A 38-year-old man with a history of a motor vehicle collision about 20 years prior to presentation which resulted in multiple left-sided rib fractures, a left-sided pneumothorax requiring chest tube placement, and a high-grade splenic laceration necessitating an emergent splenectomy that presents to outpatient pulmonary clinic for evaluation of pulmonary nodules at the request of his primary care physician. He is asymptomatic. He has a 20-pack-year of smoking history and currently smokes 6 cigarettes per day. He denies any significant exposures or recent infections. He has a family history significant for heart disease and depression, but no history of malignancy. His vital signs and physical examination are normal. He had a CT of the chest performed with representative images from the study shown in Figure 1.

A nuclear medicine scan was subsequently requested which demonstrated uptake of the technetium $99 \mathrm{~m}$-labeled sulfur colloid in the soft tissue nodules adjacent to left hemi-diaphragm (Figure 2) confirming the diagnosis of thoracic splenosis. No further treatment or diagnostic work up was required.

Splenosis is defined as auto-transplantation of splenic tissue following traumatic or surgical disruption of the spleen.

Splenosis usually occurs in the abdomen, most commonly in the left upper quadrant $(1,2)$. However, with disruption of the diaphragm in the setting of trauma, splenic tissue can migrate into the thoracic cavity, and most often settles in the inferior, posterior left pleural space (as in our patient). The diagnosis of thoracic splenosis should be suspected when one sees left basilar pleural nodules/masses in the setting of a previous trauma necessitating a splenectomy. A technetium $99 \mathrm{~m}$-labeled sulfur colloid study will demonstrate uptake of the radiotracer in the auto-transplanted splenic tissue as this radiotracer has a strong affinity for tissue arising from the reticuloendothelial system.

Gregory Gardner MD ${ }^{1}$, Kevin Breen ${ }^{1}$, Tammer Elaini MD², and Tiffany Ynosencio $\mathrm{MD}^{2}$

${ }^{1}$ Department of Internal Medicine

2Division of Pulmonary, Critical Care, Allergy and Sleep

University of Arizona College of Medicine

Tucson, AZ USA

\section{References}

1. Khosravi MR, Margulies DR, Alsabeh R, Nissen N, Phillips EH, Morgenstern L. Consider the diagnosis of splenosis for soft tissue masses long after any splenic injury. Am Surg. 2004 Nov;70(11):967-70. [PubMed]

2. Rosado-de-Christenson ML, Abbott GF. Diagnostic Imaging: Chest. $2^{\text {nd }}$ edition. Philadelphia, PA: Lippincott Williams \& Wilkins; 2012: 30-1. 